\title{
Influence of Substrate Temperature and Dopant Distribution at Two-Dimensionally Doped Superconducting $\mathrm{La}_{2} \mathrm{CuO}_{4}$ Interfaces
}

\author{
Y. Eren Suyolcu ${ }^{1}$, Yi Wang ${ }^{1}$, Federico Baiutti ${ }^{1}$, Giuliano Gregori ${ }^{1}$, Georg Cristiani ${ }^{1}$, Wilfried Sigle ${ }^{1}$, \\ Joachim Maier ${ }^{1}$, Gennady Logvenov ${ }^{1}$ and Peter A. van Aken ${ }^{1}$ \\ 1. Max Planck Institute for Solid State Research, Stuttgart, Germany
}

The discovery of novel phenomena occurring at interfaces in complex oxide structures triggered enormous interest in recent years due to the unique possibilities related to materials engineering, such as tailoring of functionalities at the atomic layer scale [1]. One of the most exciting interface effects are high-temperature interfacial superconductivity [2,3] and space-charge superconductivity [4,5], where a crucial role is played by electronic and ionic charge redistribution at the interfaces.

Atomic layer-by-layer oxide molecular beam epitaxy (ALL oxide MBE) is a unique technique for the synthesis of high-quality oxide thin films and heterostructures for fundamental studies and for the fabrication of nano-sized devices. In this work, we synthesized two-dimensionally-doped lanthanum cuprate superlattices, in which cations of single $\mathrm{LaO}$ layers in the epitaxial crystal structure are substituted by different dopants, using the unique capabilities of ALL oxide MBE. First, we discuss the effect of substrate temperature in two-dimensionally Sr-doped $\mathrm{La}_{2} \mathrm{CuO}_{4}$ (LCO) superlattices, in which a systematic decrease of the substrate temperature was carried out. Secondly, we studied the influence of dopant ionic radii on the cationic distribution in two-dimensionally doped LCO, in which differently sized dopants were employed, i.e. $\mathrm{Sr}^{2+}, \mathrm{Ca}^{2+}$, and $\mathrm{Ba}^{2+}$.

The structural and chemical investigations were conducted by scanning transmission electron microscopy (STEM) in a JEOL JEM-ARM200F STEM equipped with a cold field-emission electron source, a probe $C_{\mathrm{s}}$-corrector (DCOR, CEOS $\mathrm{GmbH}$ ), a large solid-angle JEOL Centurio SDD-type energy-dispersive X-ray spectroscopy (EDXS) detector, and a Gatan GIF Quantum ERS spectrometer. STEM imaging and electron energy-loss spectroscopy (EELS) were performed at probe semiconvergence angles of $20 \mathrm{mrad}$ and $28 \mathrm{mrad}$, respectively. Collection angles for high-angle annular dark-field (HAADF) and annular bright-field (ABF) images were 75-310 mrad and 11-23 mrad, respectively.

Structural quality of the epitaxial layers and perfect coherent interfaces of the samples are revealed via STEM-HAADF imaging (Figs. 1 and 2a). For the study of the dopant distribution, detailed EELS and EDXS analyses were performed. The dopants are found to redistribute in the out-of-plane direction (growth direction). Figure 1 shows the general view of the heterostructure revealing the structural quality of the two-dimensionally Sr-doped sample, for which the substrate temperature was decreased with $30-35{ }^{\circ} \mathrm{C}$ steps from the optimal temperature $\left(\sim 600{ }^{\circ} \mathrm{C}\right)$ [5]. The representative EELS line scan profile (red line in Fig. 1) indicates an asymmetric Sr distribution at the interfaces. Figure 2a represents the defect-free coherent interfaces of a two-dimensionally Ca-doped LCO superlattice. The atomically resolved EEL spectrum image (SI) (Fig. 2b) shows that the Ca distribution is not restricted to one atomic plane and shows similar asymmetric behavior as the two-dimensional Sr-doping. Results regarding two different effects (i.e. substrate temperature, dopant size) will be presented and correlations with the structure and the superconducting properties will be discussed. These results highlight the important role of dopant distribution in the structure and in the final interface properties [6]. 
References:

[1] Hwang, H. Y. et al, Nature Materials, 11 (2012), p.103.

[2] Gozar, A. et al, Nature, 455 (2008), p.782.

[3] Suyolcu, Y.E. et al, Microscopy and Microanalysis, 22(S3) (2016), p. 308.

[4] Baiutti, F. et al, Nature Communications, 6 (2015), p. 8586.

[5] Baiutti, F. et al, ACS Applied Materials \& Interfaces, 8 (2016), p. 27368.

[6] The research leading to these results has received funding from the European Union Seventh

Framework Program under Grant Agreement 312483-ESTEEM2 (Integrated Infrastructure Initiative I3).

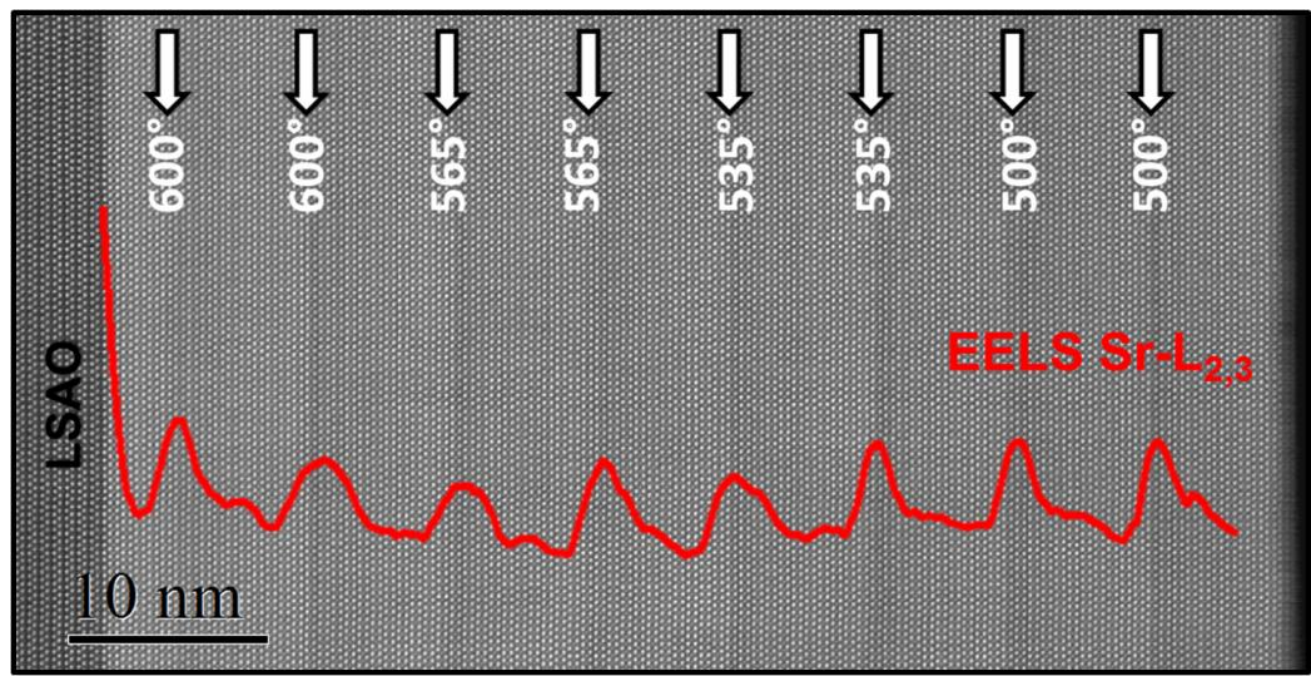

Figure 1. STEM-HAADF image of the $\mathrm{Sr} \delta$-doped LCO superlattice showing the overview of the heterostructure with revealing the structural quality. The image was taken along the [100] direction of the LSAO substrate. The inset shows the integrated Sr-L2,3 EELS line profile.
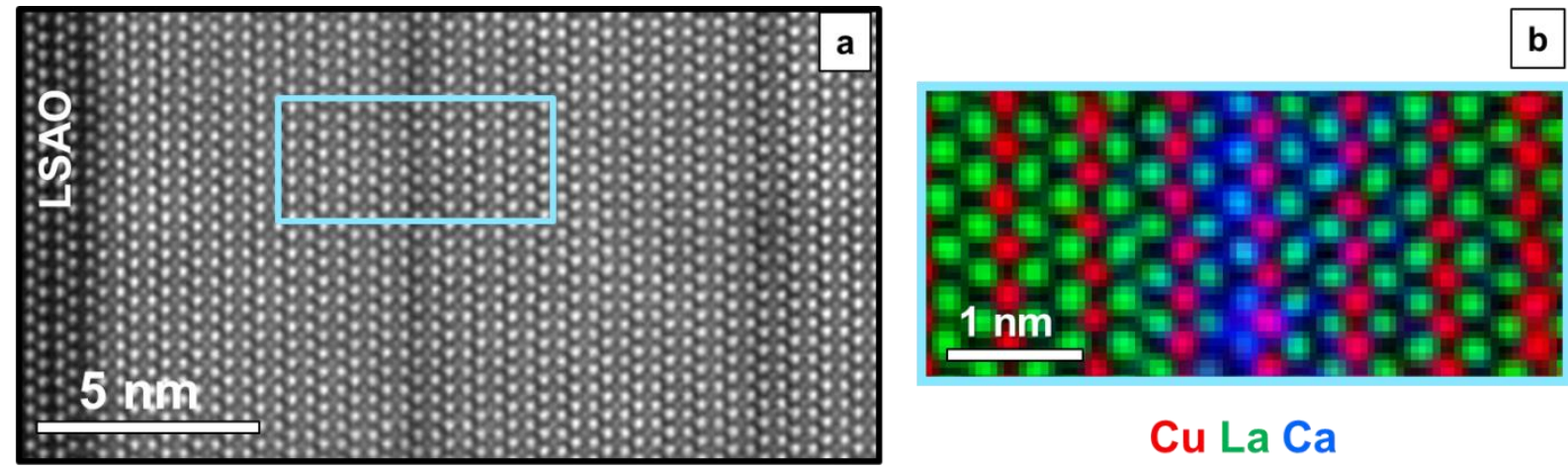

Cu La Ca

Figure 2. a) STEM-HAADF image of Ca-doped LCO superlattice showing a coherent interface and high structural quality. The image was taken along the [100] direction of the LSAO substrate. b) Atomically resolved EEL SI showing the elemental distribution of $\mathrm{Cu}$ (red), $\mathrm{La}$ (green), and $\mathrm{Ca}$ (blue). 